\title{
ANALYSIS OF THE APPLICATION OF FUZZY LOGIC METHODS FOR THE ROBOT-MANIPULATOR CONTROL SYSTEM
}

\author{
BREIDO, I. \& SHPAKOVA, L.
}

Abstract: The creation of intelligent control systems by robotic complexes is a priority for the robotics development. It is established that smart systems allow expanding the scope of robots, improving the quality of their work and reducing the operator participation in the decision-making process. In this article, we consider an example of the implementation of an intelligent control system for a robot-manipulator based on the creation of a fundamentally new control system for its electric drives and the use of linguistic variables - the theory of fuzzy logic. The work result is a fundamentally new system of intelligent control of the robot manipulator. Conclusions are made about the prospects for improving the decision making algorithm of the robot control system based on the integration of the Fuzzy control system and the theory of neural networks.

Key words: Fuzzy logic; Intelligent control system; Industrial automation; Robotic systems.
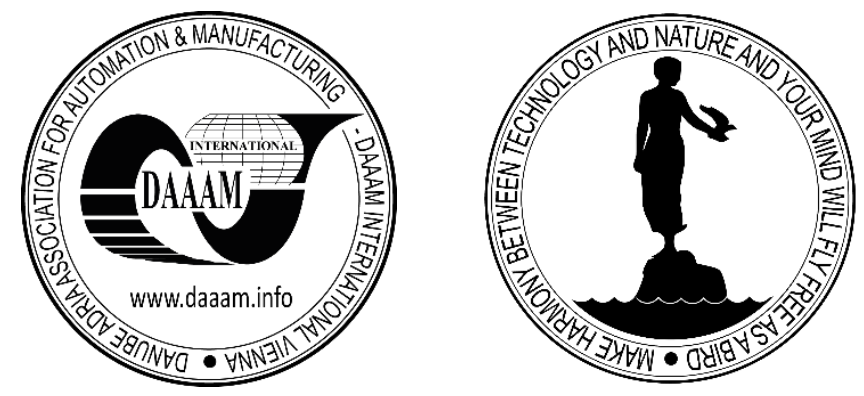

Authors' data: Univ.Prof. Dr.techn. Breido, I[ossif]*; M.Sc. Shpakova, L[yubov]**, *Karaganda State Technical University, Mira blvd. 56, Karaganda, Kazakhstan, ** Karaganda State Technical University, Mira blvd. 56, Karaganda, Kazakhstan, jbreido@mail.ru, lu.shpakova@ya.ru

This Publication has to be referred as: Breido, I[ossif] \& Shpakova, L[yubov] (2017). Analysis of the Application of Fuzzy Logic Methods for the RobotManipulator Control system, Chapter 10 in DAAAM International Scientific Book 2017, pp.121-132, B. Katalinic (Ed.), Published by DAAAM International, ISBN 9783-902734-12-9, ISSN 1726-9687, Vienna, Austria

DOI: 10.2507/daaam.scibook.2017.10 
Breido, I. \& Shpakova, L.: Analysis of the Application of Fuzzy Logic Methods for...

\section{Introduction}

The purpose of this research is to improve the efficiency and maneuver enhancement by introducing a fuzzy control unit into the control system. In the basic control system the drives were not rationally used there was a positioning error; there was no blocking of incorrect commands. The problems described above led to an unacceptably long execution of the program because of the resulting manipulation positioning error; the parts used for transportation were destroyed. To solve the posed problems the fuzzy control method was chosen as it unlike the traditional methods basically has a friendly linguistic description and is used in systems where there are many unrelated input data coordinates.

Today all robotic mechanisms are divided into three generations; each of them has limited technical and functional capabilities (Yurevich, 2005):

1) Software robots: function according to a strictly defined program;

2) Adaptive robots: have pre-programmed software, but can make changes to it (adapt) during operation;

3) Intelligent robots: they only have a general guidance on the functioning and independently make decisions based on information coming from outside.

It is possible to consider only partially the second and completely third generation of robots as intellectual. Only those systems have their own evolution of the robot program which allows not only improving the quality of its functioning, but also creating fundamentally different algorithms for interaction with the environment.

However, the third generation of robots is still a vast field for the scientists and enthusiasts' activities. While mechatronics specialists are working to create efficient and less energy-intensive designs of robots, experts in the field of artificial intelligence are occupied with the issues of creating more sophisticated mathematical algorithms for information processing and training, such as artificial neural networks.

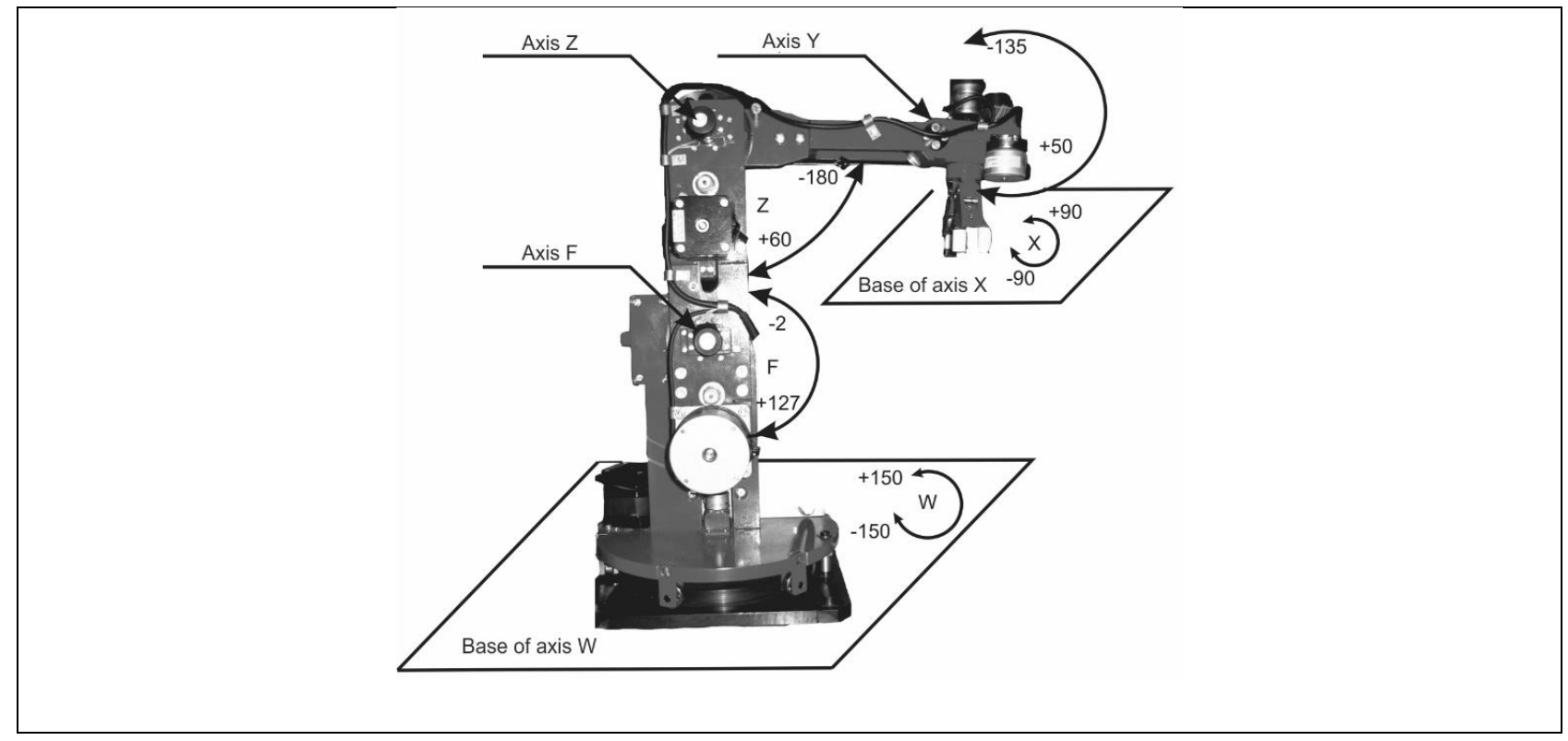

Fig. 1. Axes of rotation of the robot manipulator. 
The construction of any complex system begins with the solution of small problems, after the algorithms of which are combined, and only then it is the time of testing phase.

Works have been studied: Z. Lavic, M. Pasic, Z. Aljicevic,; A. Kostic, N. Dautbasic, M.Svaco, B. Jerbic,; I. Stiperski, D.Dlaka,; J. Vidakovic, B. Sekoranja, J.Vidakovic, B. Jerbic, F. Suligoj, C. G.Amza, D. Cicic, U. Iskakov, J. Breido which are aimed at study the mentioned fuzzy systems.

The implementation of the management system based on Fuzzy management was carried out on the training robot produced by Science and Production Institute "Training engineering and technology", the city of Chelyabinsk, South Ural State University. Figure 1 shows the rotational axes of the robot and their limiting values. The robot is designed to work as a part of laboratory stands. It consists of the following components:

1) Manipulator with a control system;

2) Power supply unit;

3) Cable communication line.

The design of the manipulators is determined, first of all, by their kinematic scheme, the type and location of the drives and the mechanisms of transferring motion from them to the manipulator links are also of great importance.

\section{Reasons for modernization of the management system}

During studying the provided software and hardware complex, the following limitations were revealed. Here are some of them:

1) The lack of the ability to set the speed for each stepper motor which does not allow speeding up the technological process;

2) The monitoring system for the limit requirements for the position of the axes. If values are exceeded then there is a failure program and the axes are not controlled;

3) The lack of the ability to perform the movement of several axes simultaneously;

4) The lack of feedback. Achieving limit values, the system comes to failure; control impulses continue to flow; the real object still stands;

5) The lack of the ability to create an executing cycle of the control program, which is to say that after executing the program, the robot stopped and to launch the ego to do the same operation, you must press the "start program" button again;

6) The lack of the ability to visualize the process on a computer monitor;

7) The lack of the ability to execute the program code in virtual mode.

\section{Analysis of research in the field of fuzzy control in robotics}

Mechatronics refers to the interdisciplinary field of engineering, which develops products whose function depends on combining mechanical, electrical and electronic components. Drives (motors) are considered as important components of all mechatronics systems. 
Stepper motors are widely used in systems requiring high accuracy in combination with fast response. Stepper motors are mainly used for simple point-to-point positioning tasks with an open-loop control.

Consequently, their operation is caused by a sequence of pulses with a predetermined time interval between consecutive pulses applied to the power rotation, and such control does not give any information about either the position of the motor shaft or the speed of rotation. Unfortunately, open-loop control systems suffer from poor performance and lack of adaptation to the load which leads to oscillatory changes in the system.

Indeed, without feedback there is no way to know when the engine missed the impulse or when the speed reaction is oscillatory nature. The principle of a closed system (feedback system) was introduced in the form of increasing the accuracy of positioning the engine, making the system less sensitive to disturbances associated with the load.

In a closed loop, the engine starts with one pulse, and subsequent pulses are generated depending on the position of the shaft and / or the speed of the motor as a result of using feedback. Today thanks to the progress made in power electronics and data processing stepper motors have become possible in closed control systems particularly for machines and automatic manipulators in spite of configuration to be used where high-precision operations are necessary.

In addition, the use of classical closed control algorithms such as proportionalintegral-differential (PID) is insufficient if the closed system is forced to adapt to the engine operating conditions. The use of fuzzy logic has a great potential in the field of artificial intelligence and mechatronics. Fuzzy logic imitates the human way of thinking and the decision-making process (Iskakov \& Breido, 2014).

\section{Definition of the requirements for the fuzzy control system of the manipulator drives}

The basis for the operation of fuzzy inference is the rule base containing fuzzy utterances in the 'If-then' form and the belonging function for the corresponding linguistic terms. The following conditions must be met:

1. There is at least one rule for each linguistic term of the output value.

2. For any term of the input value, there is at least one rule in which this term is used as a prerequisite (the left side of the rule). Otherwise, there is an incomplete base of fuzzy rules.

In general, the logical inference mechanism includes four stages: the introduction of fuzzy (phasing), fuzzy inference, composition and reduction to crisp, or defuzzification (Figure 2). 


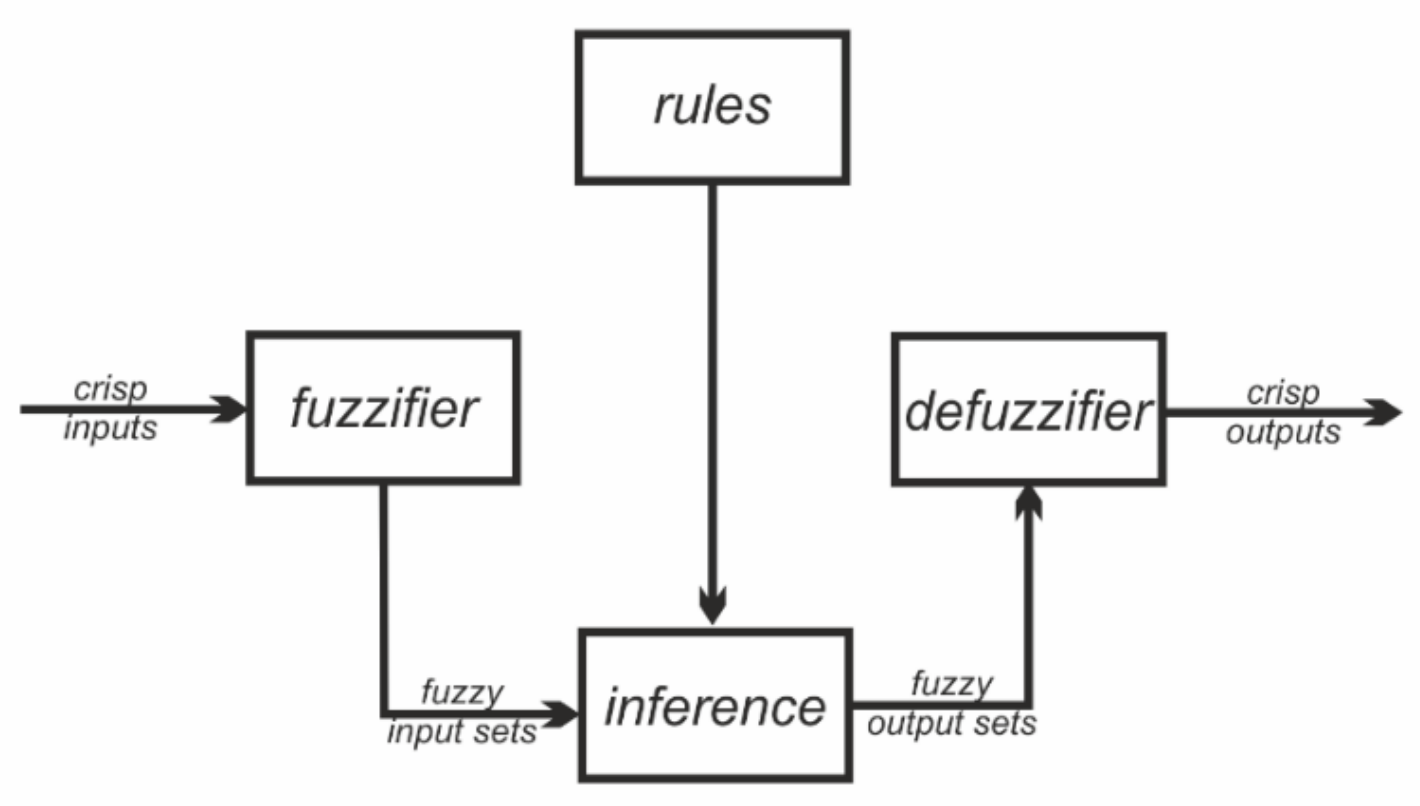

Fig. 2. Fuzzy Inference System.

Fuzzy inference algorithms differ mainly in the kind of rules used, in logical operations, and in the variety of the defuzzification methods. The models of fuzzy inference of Mamdani, Sugeno, Larsen and Tsukamoto were developed.

Fuzzification is the comparison of the set of $\mathrm{x}$ values and their relation to function $\mathrm{M}$ (x), i.e. transferring the $\mathrm{x}$ values into a fuzzy format (example with the term young).

Defuzzification is a process that is reversed by fuzzification.

All systems with a fuzzy logic function according to the same principle: the readings of the measuring instruments are fuzzy (translated into fuzzy format), defuzzificated and in the form of the usual signals are fed to the actuators.

The degree of belonging is not a probability; the distribution function is unknown, there is no repeatability of the experiments.

So if we take two mutually exclusive events from the previously considered weather forecast example: it will rain or it will not rain and will assign some ranks to them then the sum of these ranks will not necessarily be equal to 1 but if equality still exists, then the fuzzy set is considered normalized.

The values of the belonging function $\mathrm{M}(\mathrm{x})$ can be taken only from the prior knowledge, intuition (experience).

Therefore, data on the position of setting and the distance to a given point is phased and processed by Fuzzy-regulator and transferred to the defuzzificator, where the definite values of the rotation speed of direction of drives come out. The implementation of the drive control system is given below (Rezeka \& Elsodany, 2010).

\section{Creating a rule base for the fuzzy controller}

In fuzzy logic, the concept of a linguistic value is introduced, the values of which are not numbers, but the words of a natural language called terms. For example, in case 
Breido, I. \& Shpakova, L.: Analysis of the Application of Fuzzy Logic Methods for...

of control of the robot grasp, you can enter two linguistic values: DISTANCE (distance to obstacle) and DIRECTION (the angle between the longitudinal axis of the robot and the direction to the given point).

Consider the linguistic value DISTANCE. By its values, it is possible to define the terms VERY FAR, FAR, NEAR and VERY NEAR. For physical realization of a linguistic value, it is necessary to determine the exact physical values of the terms of this value. Let the value DISTANCE can take any value from the range from zero to infinity. According to the provisions of the theory of fuzzy sets, in this case each value of the distance from the specified range can be associated with a certain number from zero to one that determines the degree of belonging of a given physical distance (say $\mathrm{cm}$ ) to one or another term of the linguistic value DISTANCE. The degree of belonging is determined by called the belonging function $M(d)$, where $d$ is the distance to the interference. In our case, the distance of $7 \mathrm{~cm}$ can be determined by the degree of belonging to the term NEAR equal to 0.6, and to the term FAR - 0.4 shown in Figure 3.

Variable POSITION of grasping, which can take values in the range from 0 to $\mathrm{n}$ degrees (depending on the joint), set the terms LEFT, RIGHT, UP, BELOW, FORWARD, BACK.

Now you need to set the output values. In this example, two are enough, which will be called SPEED and STEERING DIRECTION. SPEED can contain terms: FAST, SLOWLY, MIDDLE and STOP. The output value STEERING DIRECTION can contain terms LEFT, RIGHT, DOWN, UP, FORWARD, BACK.

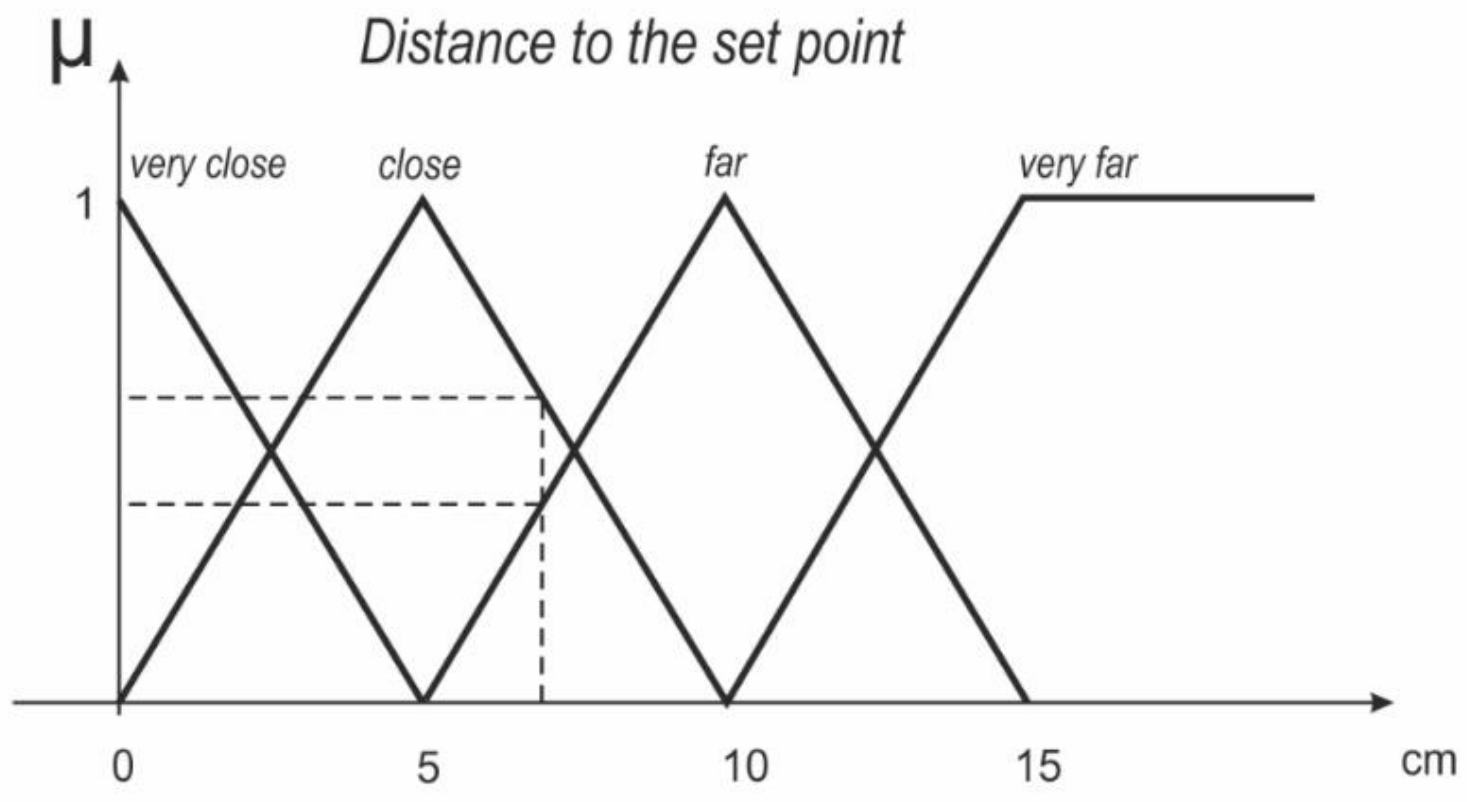

Fig. 3. Linguistic value and belonging function.

The connection between the input and output is stored in the fuzzy rule table, which is shown in Figure 4. 


\section{Distance to the set point}

\begin{tabular}{|c|c|c|c|c|}
\hline & $\begin{array}{l}\text { very } \\
\text { close }\end{array}$ & close & far & $\begin{array}{l}\text { very } \\
\text { far }\end{array}$ \\
\hline 을 & $\begin{array}{l}\text { stop } \\
\text { left }\end{array}$ & $\begin{array}{r}\text { slow } \\
\text { left }\end{array}$ & $\begin{array}{c}\text { medium } \\
\text { left }\end{array}$ & $\begin{array}{l}\text { quickly } \\
\text { left }\end{array}$ \\
\hline$\frac{2}{\frac{0}{\sigma}}$ & $\begin{array}{l}\text { stop } \\
\text { right }\end{array}$ & $\begin{array}{l}\text { slow } \\
\text { right }\end{array}$ & $\begin{array}{c}\text { medium } \\
\text { right }\end{array}$ & $\begin{array}{c}\text { quickly } \\
\text { right }\end{array}$ \\
\hline$\stackrel{\lambda}{ \pm}$ & $\begin{array}{l}\text { stop } \\
\text { down }\end{array}$ & $\begin{array}{l}\text { slow } \\
\text { down }\end{array}$ & $\begin{array}{c}\text { medium } \\
\text { down }\end{array}$ & $\begin{array}{l}\text { quickly } \\
\text { down }\end{array}$ \\
\hline .5 & $\begin{array}{l}\text { stop } \\
\text { up }\end{array}$ & $\begin{array}{l}\text { slow } \\
\text { left }\end{array}$ & $\begin{array}{c}\text { medium } \\
\text { left }\end{array}$ & $\begin{array}{l}\text { quickly } \\
\text { left }\end{array}$ \\
\hline ఏ & $\begin{array}{c}\text { stop } \\
\text { backward }\end{array}$ & $\begin{array}{c}\text { slow } \\
\text { backward }\end{array}$ & $\begin{array}{l}\text { medium } \\
\text { backward }\end{array}$ & $\begin{array}{c}\text { quickly } \\
\text { backward }\end{array}$ \\
\hline 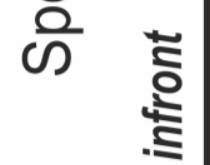 & $\begin{array}{c}\text { stop } \\
\text { forward }\end{array}$ & $\begin{array}{c}\text { slow } \\
\text { forward }\end{array}$ & $\begin{array}{l}\text { medium } \\
\text { forward }\end{array}$ & $\begin{array}{l}\text { quickly } \\
\text { forward }\end{array}$ \\
\hline
\end{tabular}

Fig. 4. Table of fuzzy rules.

Each entry in this table corresponds to its own fuzzy rule, for example, if the distance is NEAR and the RIGHT POSITION, then the STEERING DIRECTION IS SLOWLY LEFT.

That is why the crasp of a robot with fuzzy logic will work according to the following principle: the data from the sensors about the distance to the target and the direction to it will be fuzzy, processed according to the table rules, defuzzificated and the received data in the form of control signals will be sent to the robot drives.

\section{Implementation of the robot manipulator control system by the methods of fuzzy logic}

The combined procedure for determining of the belonging function of the fuzzy rule "IF-THEN" and the aggregation of several such rules is called an inference procedure or an inference.

In our case, the entire previously presented rules base can be in the form presented in Figure 5. 
Breido, I. \& Shpakova, L.: Analysis of the Application of Fuzzy Logic Methods for...

Where position is position ( $\mathrm{P}$ is positive, $\mathrm{N}$ is negative), distance is distance ( $\mathrm{ZE}$ is very near, PM is near, PB is far away, PBB is very far). And the output values ST stop, $\mathrm{SN}$ - slowly negative, $\mathrm{SP}$ - slowly positive, $\mathrm{MN}$ - medium negative, MP - medium positive, QN - quickly negative, QP - quickly positive.

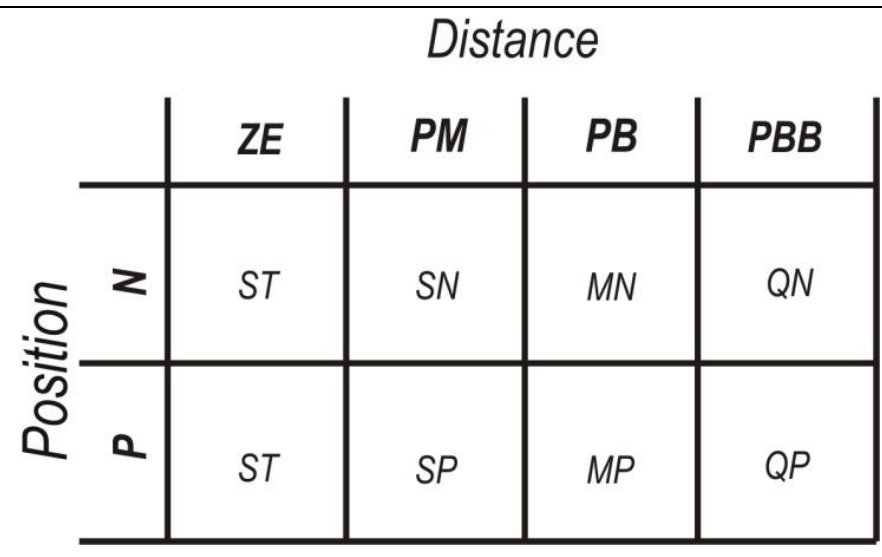

Fig. 5. Table of rules for the robot manipulator.

Aggregation, or the composition of several "IF-FROM" rules, connected by the "OR" conjunction, is carried out by maximizing the OP of all combined rules.

In the implementation of the inference procedure, the maximin method is most often used, in which a limited amount of terrier and artistic information is obtained by the trapezoid.

In order for the result of belonging function to find a specific value of the control action, a defuzzification procedure is used. The most widely used method is the "centre of gravity", according to which the value of the y-control effect is found as the abscissa of the "centre of gravity" of the area, of under the AF plot.

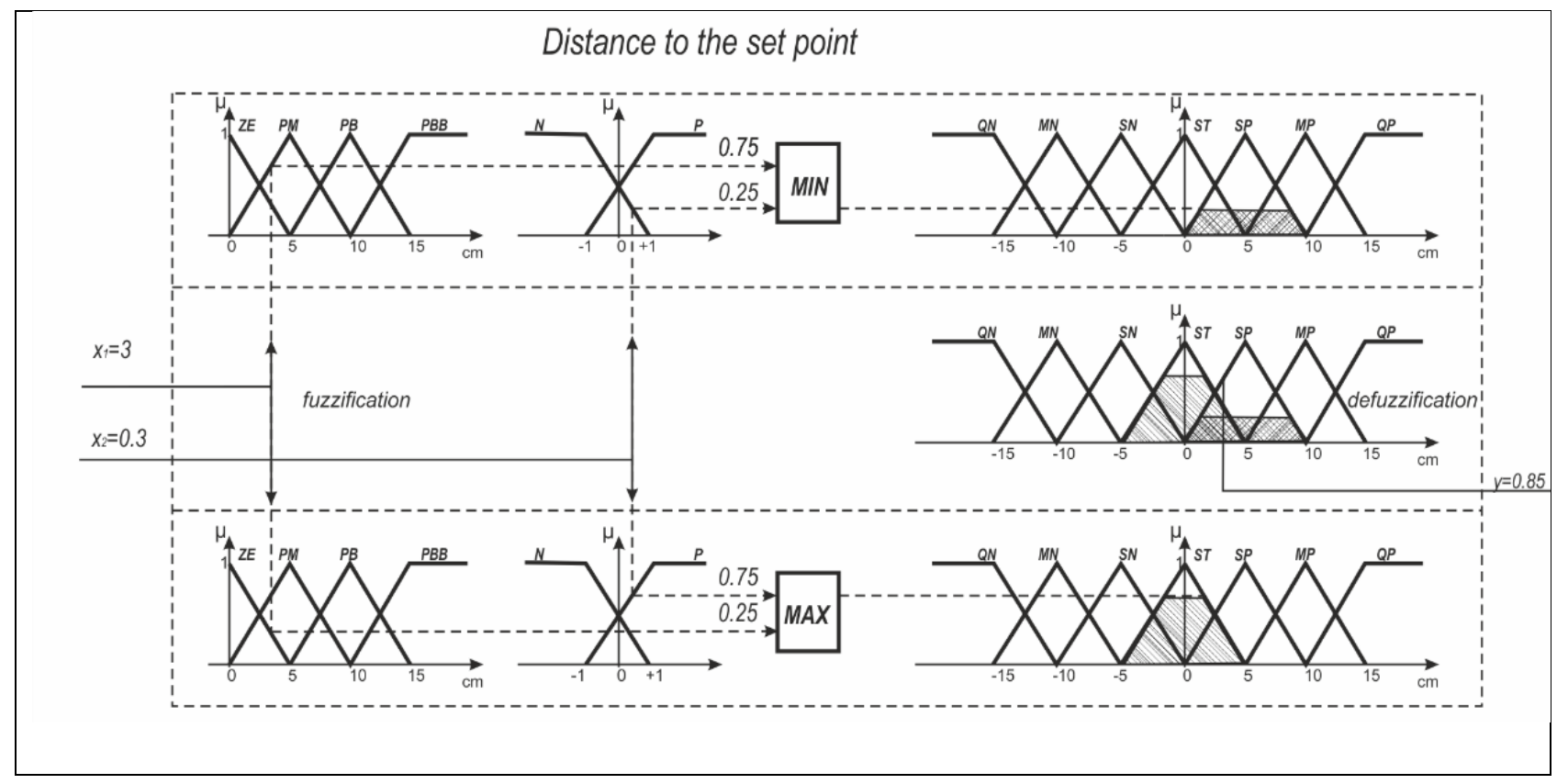

Fig. 6. Implementation of fuzzy logic procedures for two control rules. 
Figure 6 illustrates the basic phasing-logic procedures for the two fuzzy IF-THEN rules for the robot manipulator and contains two fuzzy values $\mathrm{x} 1$ и х 2 (distance to the point and position respectively) connected by the AND and OR conjunctions.

We illustrate the fuzzy logic procedures for the robot manipulator, in which the input values $\mathrm{x} 1=3$ and $\mathrm{x} 2=0.3$. The fuzzy control algorithm is represented by the following four rules:

Rule 1: IF PM and P then SP

Rule 2: IF PM and N then SN

Rule 3: IF ZE or P then ST

Rule 4: IF ZE or N then ST

Executing the aggregation procedure, we obtain the resulting belonging function corresponding to the aggregated functions. Found as a result of defuzzification by the method of the gravity center $y=0.85$.

The results of the work are presented in Figures $7-10$.

Figure 7 shows the graph of the achievement of the limit values of the previous control system. The abscissa axis is the mechanical limit. The sending of control pulses occurs at a frequency of $20 \mathrm{~ms}$. When the limit value is reached, the control action continues but the operation stops which causes the failure system.

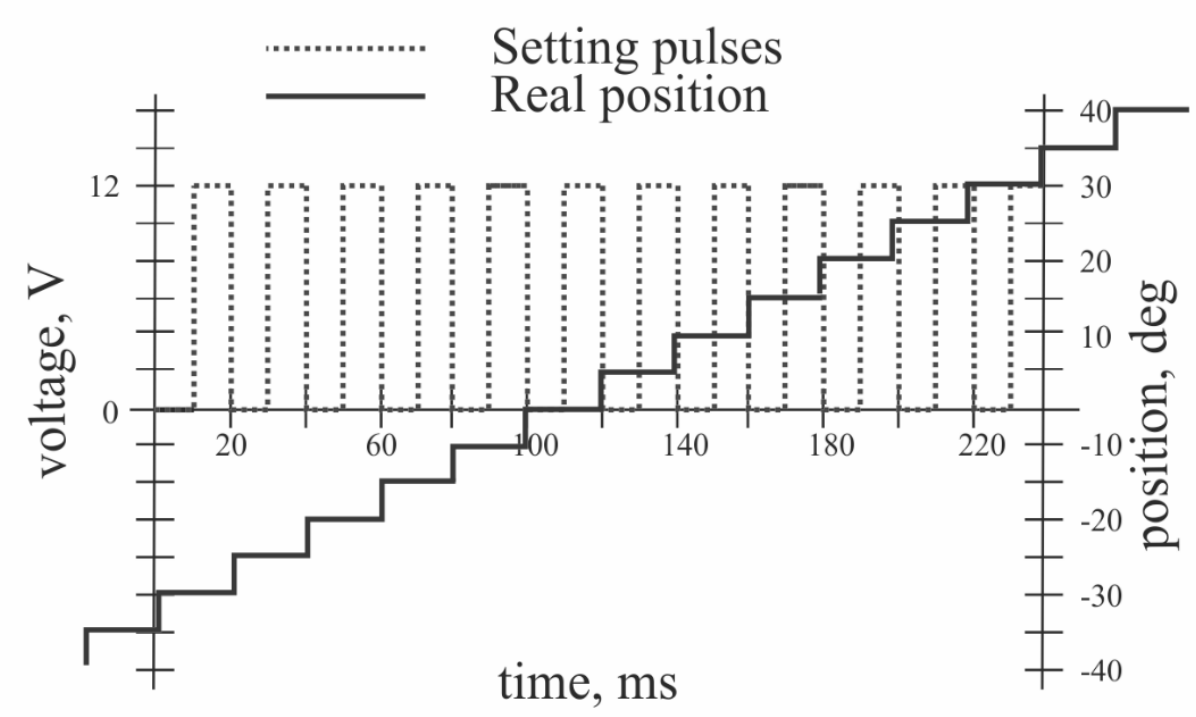

Fig. 7. Graph for limit values reaching of the previous control system.

Figure 8 shows the graph of achievement of limit values with the help of a fuzzy controller looks like this: at the moment of reaching the limit the sending of the control action stops.

Figure 9 shows the approximation of the PM to a given point from -0 degrees to +10 . And it with the help of a fuzzy controller is shown in Figure 10. 


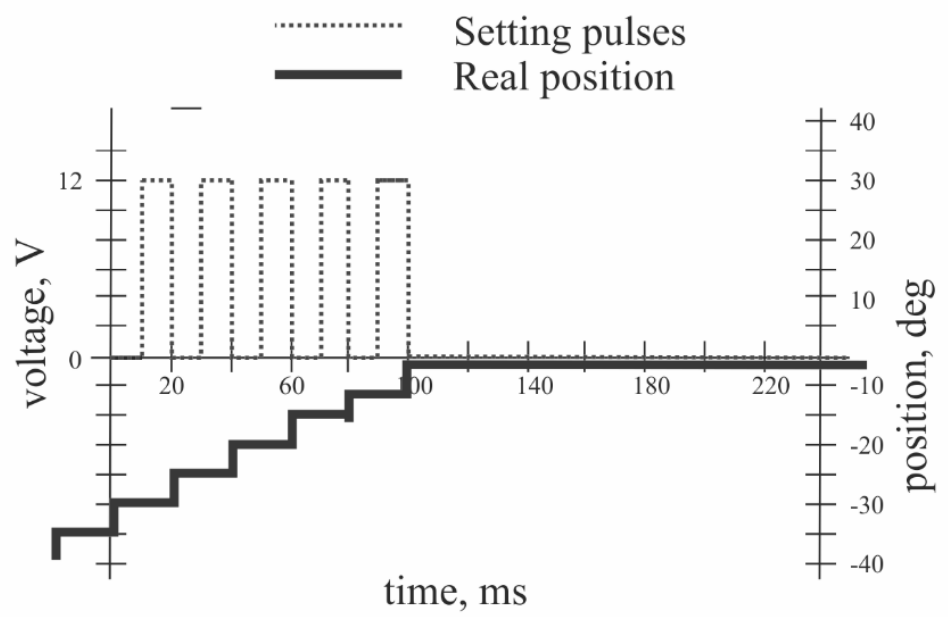

Fig. 8. The graph of the mechanical limit achievement by the new control system.

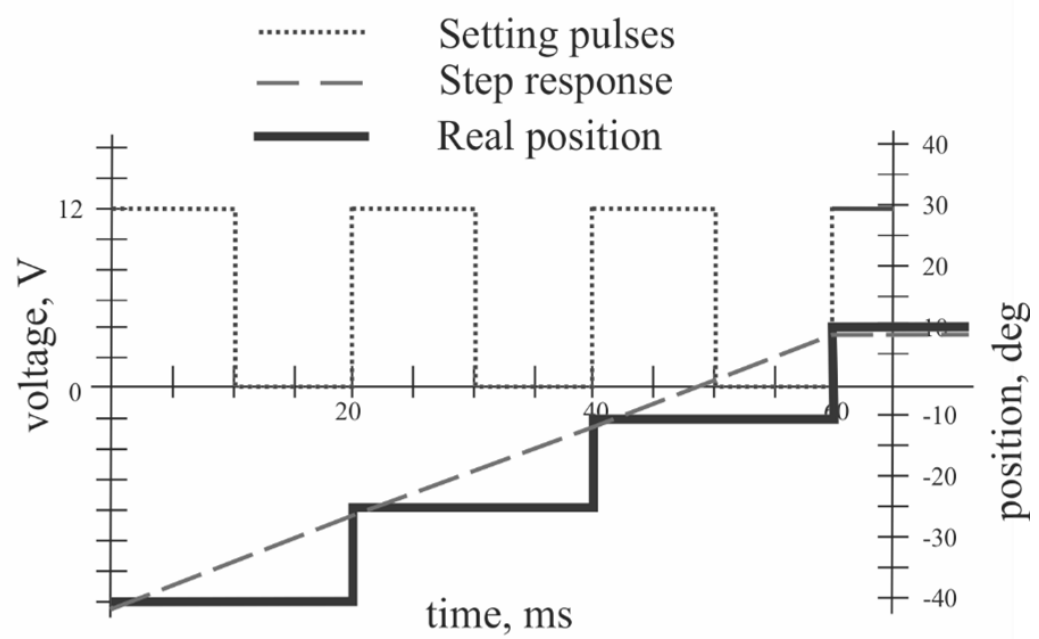

Fig. 9. Graph of axis rotation from -40 to +10 degrees using the old control system.

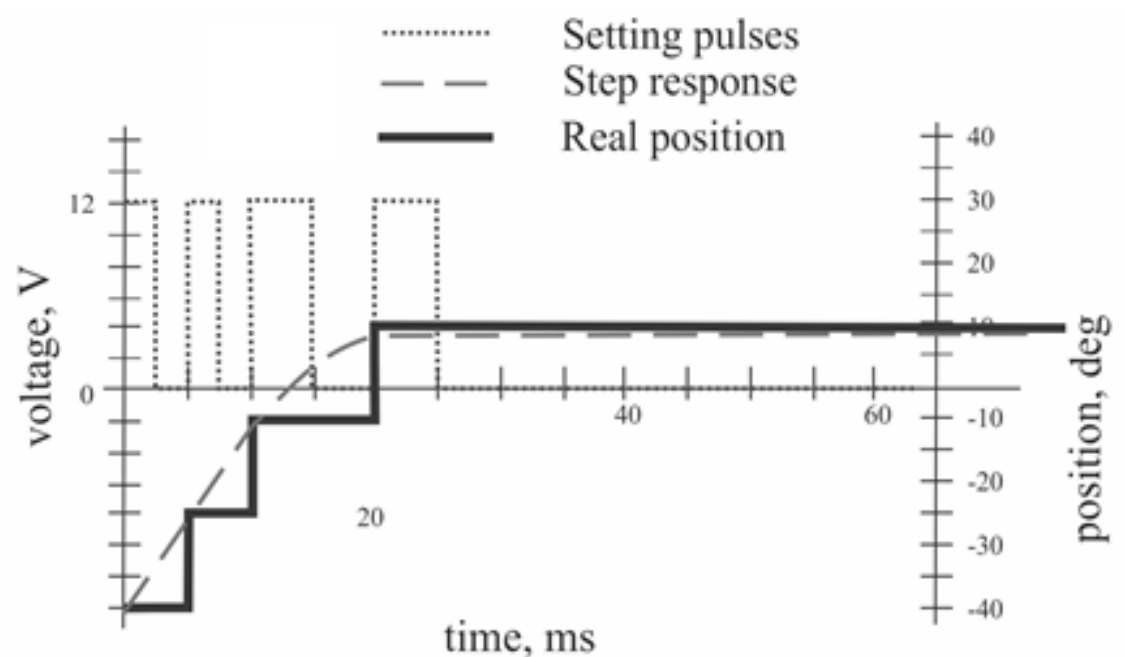

Fig. 10. Graph of axis rotation from -40 to +10 degrees with fuzzy controller. 
Comparing, it can be seen that the speed of access time with fuzzy control increased from $60 \mathrm{~ms}$ to $20 \mathrm{~ms}$ due to the consecutive sending of pulses with high frequency and slowing down as we approached the given point.

\section{Prospects for the development of the system}

Analysing the graphs we find that fuzzy control allows blocking incorrect commands by stopping the supply of control pulses to stepper motors and accelerating the process of reaching the grasping specified point by forming control pulses depending on the distance to a given point.

Summarizing the results, we can say that fuzzy systems have several advantages:

1. The ability to represent the inherent uncertainty of human knowledge with linguistic values;

2. Simple interaction of the domain expert with the system engineering engineer;

3. Easy interpretation of results due to the presentation of natural rules;

4. Easily expand the knowledge base by adding new rules;

5. Reliability of a possible disorder in the system.

6. However, fuzzy systems also have disadvantages:

7. Failure to generalize the exclusively prescribed rules in the database;

8. Instability to topological changes in the system, such changes will require changes in the rules base;

9. Dependence on the presence of an expert to determine the logical rules;

Further development of the object of research involves the introduction of an input variable, by adding to the existing object of a technical vision system.

In order to solve the shortcomings of management based on fuzzy logic implementation it is supposed the development of neuro-fuzzy systems.

Neural networks are trying to form the biological functions of the human brain. This leads to the idealization of neurons as discrete units distributed processing. Its local or global connections within the network are also idealized so leading to the abilities of the nervous system.

Neuro-fuzzy term means a type of system characterized by a similar fuzzy controller structure, where fuzzy sets and rules are corrected by configuring neural networks using iterative vector data methods (Input and Output System Data).

The combining reasons for these two paradigms come from the difficulties and inherent limitations of each isolated paradigm. This type of system is characterized by a fuzzy system, where fuzzy sets and fuzzy rules are adjusted using input output information (Lucas, 2000).

\section{Conclusion}

In the robot manipulator provided for modernization there were problems with the development of user commands by means of insufficient description of working areas and irrational use of system drives. The solution of the problems described above is possible only with a complex change in the control system. For the study the method 
of Fuzzy regulation was chosen as one of the modern methods of regulation which differs significantly from traditional methods of regulation.

The result of the implementation of the fuzzy controller showed that the method can significantly improve the accuracy and speed of the robot manipulator which is shown in Figures 8 and 9.

As a result of studying the work in the field of fuzzy regulation, it was revealed that this method is increasingly used in neural-fuzzy systems (neural fuzzy control systems). Further work will be related to the addition to the existing regulatory system based on neural networks.

\section{References}

Aljicevic, Z.; Kostic, A.; Dautbasic, N. \& Karli, G. (2016). Model of Fuzzy Logic for Selection Infrastructural Investment Project of Wind Farm Locations In Annals of DAAAM for 2016\& Proceedings of the 27th International DAAAM Symposium. Vienna: DAAAM International. ISBN 978-3-902734-08-2, ISSN 1726-9679

Amza. C. G. \& Cicic. D. T. (2014). Industrial Image Processing Using Fuzzy-Logic In Annals of DAAAM for 2014\& Proceedings of 25th International DAAAM Symposium. Vienna: DAAAM International.

Iskakov. U. \& Breido. J. (2014). Development of Control Algorithm for Adaptive Leakage Current Protection Devices' Using Fuzzy Logic In Annals of DAAAM for 2014\& Proceedings of 25th International DAAAM Symposium. Vienna: DAAAM International. ISBN 978-3-901509-73-5. ISSN 1726-9679.

Vieira J.; Morgado Dias F. \& Mota A. (2004). Neuro-Fuzzy Systems: A Survey. WSEAS Transactions on Systems, Issue 2, Vol. 3, pp. 414-419.

Lavic, Z.; Pasic, M.; Vucijak, B. \& Dukic, N. (2016). Fuzzy Logistics Multicriteria Decision Making Model for Facilities Layout In Annals of DAAAM for 2016\& Proceedings of the 27th International DAAAM Symposium. Vienna: DAAAM International. ISBN 978-3-902734-08-2, ISSN 1726-9679

Lucas A.V. (2000). Fundamentals of fuzzy-control. Textbook for university students studying in the field of "Automation and management" - Ekaterinbur. 62pp

Rezeka S.F.; Elsodany N.M. \& Maharem N.A. (2010). Fuzzy gain scheduling control of a stepper motor driving a flexible rotor. European Journal of Scientific Research. Svaco, M.; Jerbic, B.; Stiperski, I.; Dlaka, D.; Vidakovic, J. \& Sekoranja, B. (2016). T-Phantom: a New Phantom Design for Neurosurgical Robotics In Annals of DAAAM for 2016\& Proceedings of the 27th International DAAAM Symposium. Vienna: DAAAM International. ISBN 978-3-902734-08-2, ISSN 1726-9679

Vidakovic, J.; Jerbic, B.; Suligoj, F.; Svaco, M. \& Sekoranja, B. (2016). Simulation for Robotic Stereotactic Neurosurgery In Annals of DAAAM for 2016\& Proceedings of the 27th International DAAAM Symposium. Vienna: DAAAM International. SBN 978-3-902734-08-2, ISSN 1726-9679

Yurevich E.I. (2005). Fundamentals of robotics. The 2-nd edition. St. Petersburg "BHV-Petersburg". 